\title{
ALGUNOS Ascomycetes Y Basidiomycetes INTERESANTES DE CHILE
}

\section{(Some interesting Ascomycetes and Basidiomycetes of Chile)}

\author{
Waldo Lazo, A. \\ Departamento de Ciencias Ecológicas, Facultad de \\ Ciencias, Universidad de Chile, Santiago, Chile.
}

Palabras clave: Ascomycetes, Basidiomycetes Key words: Ascomycetes, Basidiomycetes

\section{RESUMEN}

La mayoria de las especies füngicas nativas de Chile son poco conocidas, algunas de ellas, notables por el aspecto de sus carpóforos, nunca se han publicado ilustraciones(Lazo, 1984). En esta publicación se describen e ilustran 11 especies interesantes, con un breve comentario acerca de los hongos comestibles yvenenosos más importantes del pais.

Entre las especies nativas comestibles, se incluye a: Cyttaria espinosae Lloyd, Cyttaria berteroi Berk (nombre común "pinatra", "piñatra", dihueñe"); Fistulina hepatica Schaef. ex Fr. var. antarctica (Speg.) Wright (n. común "lengua de buey", crece sobre madera muerta); Boletus loyo Phil ex Speg. (n. común "loyo"); Ramaria valdiviana Sing (n. común "changle"); Grifola gargal Sing. (n. común "gargal", crece sobre madera); Armillariella montagnei Sing. (n. común "pique", crece sobre madera). Las fructificaciones de las Cytarias son consumidas crudas por lo general, costumbre imprudente, si se tiene en cuenta que en otros hongos en estado crudo, se han descrito compuestos cancerigenos (Toth, 1986). A las fructificaciones del hongo introducido Ramaria flava (Fr.) Quél., también se les llama "changles" y al igual que $R$. valdiviana, esta especie se desarrolla en el suelo en bosques de la zona austral.

Garrido (1985), cita 28 hongos tóxicos en Chile. De ellos, han ocasionado micetismos mortales Lepiota locaniensis Espinosa, la cual al parecer es una especic ya extinguida (Simonetti \& Lazo, 1994), Amanita toxica (Lazo) Garrido \& Bresinsky, de amplia distribución en bosques y potreros en las zonas central -austral. Además. Amanita phalloides (Vaill. ex. Fr.) Secr. (Valenzuela et al.,1992)

De "Hongos de Chile". Atlas Micológico (Lazo inédito) se ha tomado 11 interesantes especies que se describen e ilustran a continación.

1.- Gloeosioma vitellina (Lev.) Bres. Ann. Mycol. 18: 51.1920.

\section{SUMMARY}

Most of native fungal species of Chile are scarcely known. Some of them, remarkable for their aspect, color illustrations have never been pubblished (Lazo 1984). Eleven interesting species are described and illustrated in this issue. A brief comment on the most important edible and poisonous fungi in the country is also enclosed.

Receptáculo cupuliformes de 30-60 mm de diam., anaranjado en su cara externa, anaranjado blanquecino en la cara interna, liso, gelatinoso. Esporada rosado salmón. Esporas de 23.8-34.5 x 20-23.7 $\mu \mathrm{m}$, hialinas, espinulosas, elipsoides, con un corto pedicelo. Parásito de Nothofagus y otros arboles. Especie no comestible. Chile austral.

2.- Neoclitocybe byssiseda (Bres.) Sing. Sydowia 15: 55.1961 .

Píleo embudiforme de 30-60 $\mathrm{mm}$ de diámetro. Lamelas decurrentes. Estípite parejo, con un micelio sedoso basal que cubre el sustrato sobre el cual se desarrolla. Esporas 6-6.7 x 2.5-3 $\mu \mathrm{m}$, hialinas, lisas, cilíndricas. Se desarrolla sobre madera semipodrida.Especie no comestible. Chile central.

3.- Ramaria flaccida (Fr.) Ricken var. chilensis Lazo, Mycologia 64: 77-787 1972.

Basidiocarpos de 70-100 mm de altura, ocráceos a parduzcos, muy ramificados. Esporas de 4-6 $\times$ 2.5-4 $\mu \mathrm{m}$, parduzcas, verrucosas, elipsoides. Se desarrolla en el suelo entre los restos vegetales. Es la Clavariacea más abundante en Chile central y sus diferencias con $\boldsymbol{R}$. flaccida son tan significativas que merece ser descrita, no sólo como una variedad de $R$. flaccida, sino como una especie distinta (Ramaria chilensis). Especie no comestible y de sabor acre. 1972.

4.- Geastrum jurei Lazo, Mycologia 64: 768-798.

Gastrocarpo abovedado. Exoperidio de $26 \mathrm{~mm}$ de anchura y $15 \mathrm{~mm}$ de altura, rasgado en 5 rayos agudos, la 
superficie externa con abundante incrustaciones de arena y desperdicios. Pedicelo de color crema, comprimido, glabro. Endoperidio de $9 \mathrm{~mm}$ de ancho y $8 \mathrm{~mm}$ de alto, redondo, glabro. Peristoma indefinido, aunque debido a su color se lo distingue facilmente del endoperidio, cónico, sedoso, arrugado radialmente, grisáceo parduzco. Esporas de 5-5.5 $\mu \mathrm{m}$, pardo rojizas, globosas, con verrugas cortas e irregulares.Especie no comestible. Chile central.

\section{8: 51912}

5.-Boletus loyo Phil. ex Speg., An. Mus. Nac. B.A.

Píleo de 80-120 mm de diámetro, rojizo a granate, semiconvexo, globoso, seco. Tubos amarillos que toman un color azulado al magullarlos Esporada olivácea. Estípite rosado - rojizo con micelio basal blanquizco. Esporas de $14-15 \times 5 \mu \mathrm{m}$ parduzcas, lisas, fusiformes. Desarrollo en el suelo. Comestible. Chile central y austral.

6.-Gerronema versatile (Bert. \& Mont) Sing., Mycof. Austr.: 51. 1969.

Píleo de 19-22 mm de diam., pardo muy claro a blanquizco, cóncavo a umbilicado, ondeado, transparentemente estriado. Lamelas crema rosadas, algo decurrentes. Estípite concoloro, parejo a veces excéntrico. Esporas de 8-9 (11) x 4-5 (6) $\mu \mathrm{m}$, hialinas, inamiloides, lisas, elipsoides. Se desarrolla en los espacios abiertos entre el pasto de los potreros. Especie no comestible. Chile central.

7.- Mycena chusqueophila Sing. in Sing. \& Moser, Mycopathol. Mycol. Appl. 26: 159. 1965

Píleo de 4-7 mm de diam., amarillo, seco, convexo, surcado, lamelas amarillentas, adnadas, estrechas. Estípite de 10-35 $\times 0.2-0.3 \mathrm{~mm}$, amarillo-anaranjado, seco, glabro. Contextura delgada. Esporas de $7.2-8.8 \mu \mathrm{m}$, hialinas, debilmente amiloides, lisas, elipsoides. Crece sobre Chusquea sp. muerta. Especie no comestible. Chile austral.

8.- Plectania chilensis (Mont.) Gamundi. Bol. Soc. Arg. Bot. 13: 270-273.

Apotecios de 35 a $80 \mathrm{~mm}$ de diam., cupuliformes, pardo negruzcos, casi negros, la superficie externa de aspecto aterciopelado. Himenio liso en estado fresco. Estípite de 5-25 $\times 5-10 \mathrm{~mm}$, concoloro, venoso. Contextura gelatinosa. Esporas de 25-32 x 8.8-10.8 $\mu \mathrm{m}$, hialinas, fusoideo-asimétricas. Ascos de $380-430 \times 18-20 \mu \mathrm{m}$, cilíndricos, angostados en la base. Crece sobre madera semipodrida y en tocones ocasionalmente cubiertos por musgos, a veces parcialmente enterrados en el suelo. Especie no comestible. Chile central y austral. 1953.

9.- Pholiota baeosperma Sing., Sydowia 7: 217.

Píleo de 30-45 $\mathrm{mm}$ de diam., amarillo ocráceo, plano, convexo, con cubierta glutinosa. Lamelas blan100 quizcas, adnexadas. Estípe blanquizco, hueco, a veces con un tomento micelial blanco en la base. Esporada parda. Esporas de $6.5 \times 4 \mu \mathrm{m}$, ocráceo-parduzcas, elipsoides. Se desarrolla en el suelo en los prados y arboleadas entre los restos vegetales. Especie no comestible. Chile central.

10.- Psilocybe argentina (Speg.) Sing., Mycofl. Austr.: 241. 1969.

Píleo de 12-15 mm de diam., parduzco-dorado, con el margen y la mitad inferior con marcadas estrías pardas, semigloboso, convexo, glabro. Estípite concoloro. Esporada casi negra. Esporas de 13.5-15 × 8.5-9 × 7-8 $\mu \mathrm{m}$, fuscas lisas, elipsoides. Se desarrolla sobre estiércol bovino o equino. Especie toxica. Chile central y austral.

11.- Cyttaria berteroi Berk., Trans. Linn. Soc. London 19: 41. 1841

Fructificación de 40-90 mm de altura, 40 a $110 \mathrm{~mm}$ de largo y $25-45 \mathrm{~mm}$ de grosor, blanca, turbinada lijeramente comprimida a globosa cuando nueva. Superficie suave, lisa con surcos exteriores que limitan las bocas de los apotecios. Al practicar una sección longitudinal se comprueba que la pulpa es resistente, de color anaranjado y recorrida por nerviaciones blancas. Apotecios de 7-11 $\mathrm{mm}$ de diam. Himenio de intenso color anaranjado. Esporas de 11-19 $\mu \mathrm{m}$ de diam., globosas, de color oliváceo. Ascos de 200-260 $\mu \mathrm{m}$ de longitud, cilíndricos, atenuados en la base. Sabor agradable. Comestible. Chile austral y central.

\section{REFERENCIAS}

Garrido, N.( 1985). Index Agaricalium Chilensium. J. Cramer.Vaduz.

Lazo, W. (1983). Introducción al estudio de los hongos superiores II. Bol. Micol. 1: 77-119

(1984). Introducción al estudio de los hongos superiores III. Bol. Micol. 2: 27-66

(inédito). Hongos de Chile. Atlas Micológico.

Simonetti, J. A. \& Lazo, W.(1994). Lepiota locaniensis: an extinct chilean fungus. Rev. Chil de Hist. Nat. 67: 351-352

Singer, R. (1969). Mycoflora Australis. Beih. Nova Hedwigia 29:1-406

Spegazzini, C. (1910). Fungi Chileansis. Contribución al estudio de los hongos chilenos. Librería Nacional. Buenos Aires.

(1921). Mycetes Chilensis. Bol. Acad. Nat. Ci: 25: 1-124

Toth, B. \& Erikson, J. (1986). Cancer Induction in myce by feeding of the uncooked cultivated mushroom of commerce.Agaricus bisporus. Ca. Res. 46: 4007- 4011

Valenzuela, E.; Moreno, G. \& Jeria, M. (1992). Amanita phalloides en bosques de Pinus radiata de la IX región de Chile: Taxonomia, toxinas, métodos de detección, intoxicación faloidiana. Bol. Micol. 7 : 17-21 

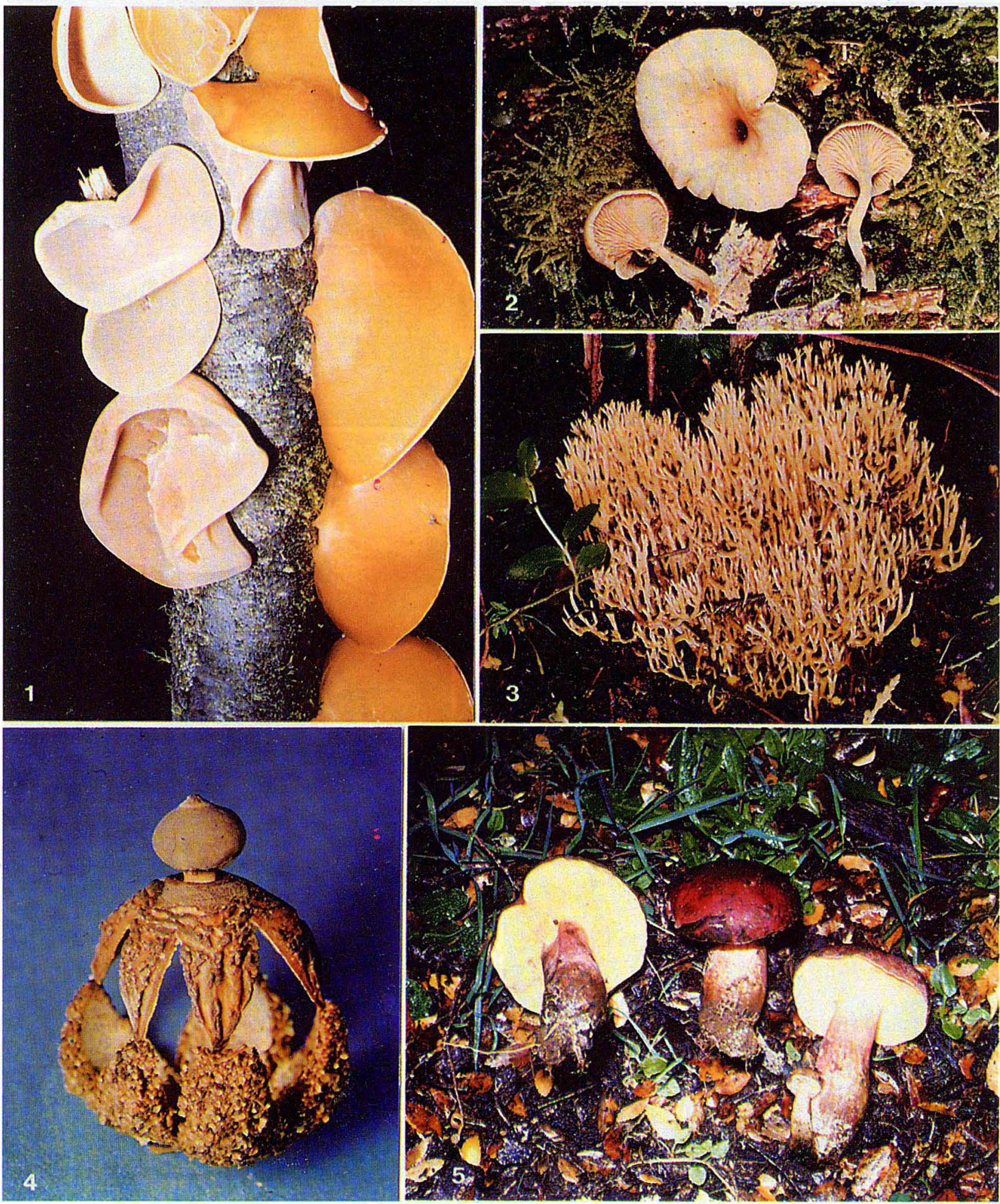

Figuras (1-11). 1.- Gloeosoma vitellina. 2.- Neoclitocybe byssiseda. 3.- Ramaria flaccida var. chilensis. 4.Geastrum jurei. 5.- Boletus loyo. 6.- Gerronema versatile. 7.- Mycena chusqueophila. 8.- Plectania chilensis. 9.- Pholiota baeosperma. 10.- Psilocybe argentina. 11.- Cyttaria berteroi. 


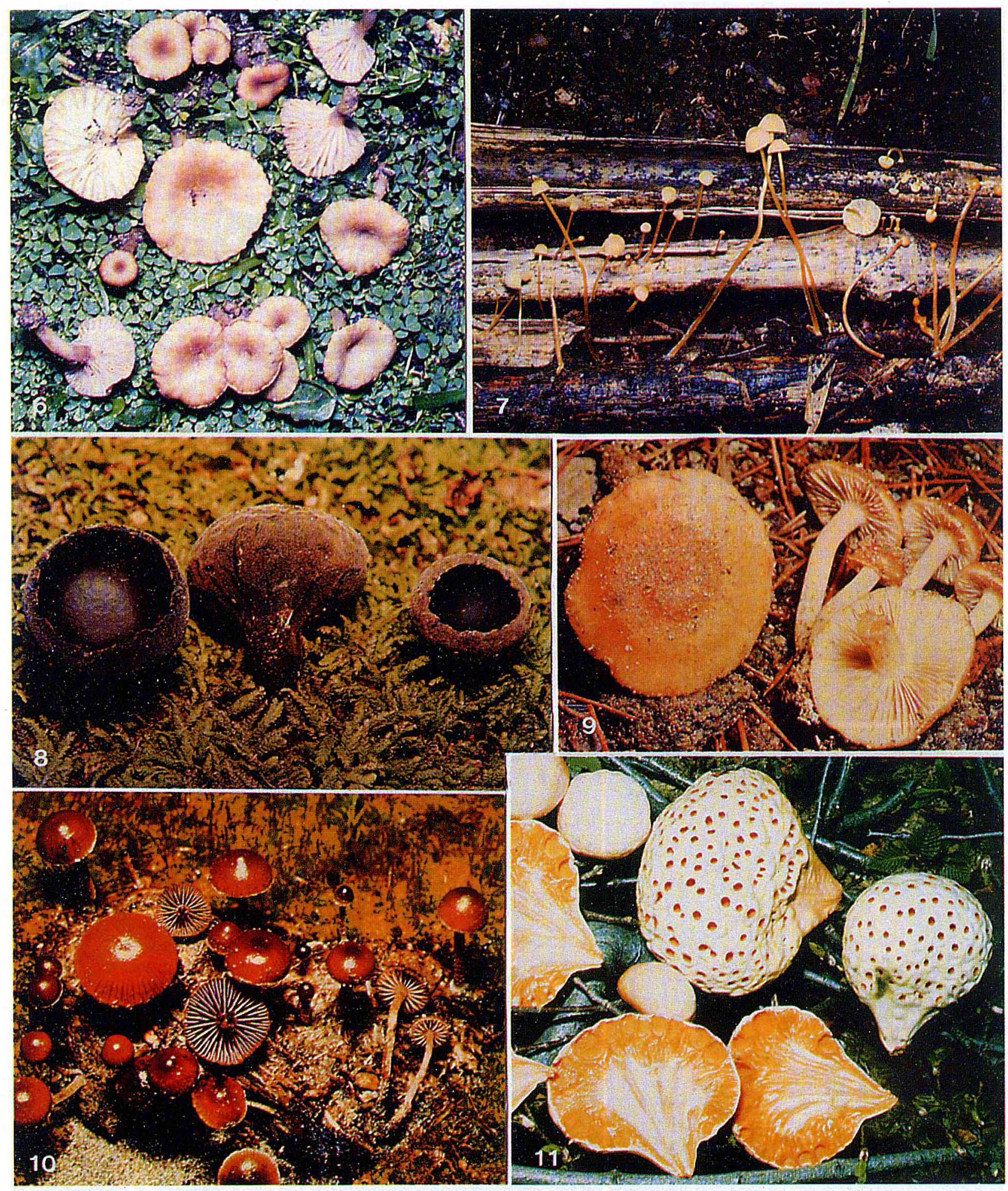

